\title{
Childhood tuberculosis in developing countries: prospects for improved diagnosis and control
}

\author{
$A$ vaccine more effective than $B C G$, and better diagnostic tests are urgently needed
}

Childhood tuberculosis (TB) has rightly been called "a hidden epidemic" [1]. Although a high burden of disease exists, documenting TB disease burden in children in low income countries has been particularly challenging, resulting in the problem of childhood TB in highly endemic countries to be largely ignored by the international and local public health communities. Global control efforts by agencies such as the World Health Organization (WHO) and by national tuberculosis control programmes have focused on notification of sputum acidfast bacillus (AFB) positive cases and directly observed therapy (DOTS) for those who are smear-positive as they are most infectious. However, this strategy overlooks childhood TB because only a small proportion of children with pulmonary TB have sputum smears positive for AFB, and case definitions for pulmonary TB in children are lacking [2]. Additionally, children suffer a higher proportion of extra-pulmonary tuberculosis compared to adults [2]. In practice, bacteriological diagnosis to confirm TB in children with suspected disease is rarely attempted in developing countries, and only rough estimates of disease burden can be derived. By some calculations, estimated TB case rates among children are in the range of 100 to 200 per 100000 children less than 15 years of age in several high burden countries such as Afghanistan, Pakistan, Kenya, South Africa and Zimbabwe [2].

Several international organisations including the International Union Against Tuberculosis and Lung Disease (IUATLD), WHO, International Paediatric Association and the US-based Centers of Disease Control and Prevention co-sponsored a workshop in October 2002 to review gaps in knowledge about childhood TB and assess research needs. The consensus and recommendations from this workshop have recently been published [3]. Briefly, the experts concluded that control of childhood TB in developing countries warrants substantially increased effort and commitment for a number of reasons. First, available evidence suggests that childhood TB is a significant contributor to child morbidity and mortality in countries with a high burden of the disease. Second, information on the burden of both TB disease and infection with Mycobacterium tuberculosis among children can serve as useful indicators of TB transmission, and the impact of control programmes. Third, in some highburden countries such as Pakistan and Afghanistan, children under the age of 15 years may represent a substantial case burden, accounting for up to $25 \%$ of all TB diseases because of the combination of high population growth rates $(50 \%$ of the population being under the age of 15 years), and high rates of TB transmission and malnutrition $[3,4]$. Fourth, children with untreated latent TB represent the future reservoir of $\mathrm{TB}$ disease that will hinder TB control efforts for decades to come. Finally, many large and well documented school outbreaks have shown that older children and adolescents are capable of transmitting TB to others and represent a pool of infectious individuals who must be diagnosed and treated appropriately [5-8].

\section{Natural history of childhood tuberculosis}

Much is known about the natural history of childhood TB from the pre-chemotherapy era and from cohort studies of children exposed to AFB smear-positive adults $[9,10]$. Household exposure to a sputum smearpositive case for several months results in $60-80 \%$ of children acquiring infection as measured by a tuberculin skin test (TST) reaction [10]. Progression to TB disease depends on several risk factors such as age of the child, nutritional status, and co-morbid conditions such as HIV infection. Without prophylactic therapy, $40-50 \%$ of infants and $15 \%$ of older children with infection will develop the disease over the next two years $[10,11]$. The progression from infection to disease is often a continuum and the distinction between infection and disease in children is not always clear. Also, many children with radiological abnormalities are clinically asymptomatic.

Infection starts with a Ghon complex in the lung parenchyma and regional lymph nodes. In most children this resolves spontaneously with some residual calcification or scarring. But some children, particularly infants and children less than 4 years of age, develop hilar or paratracheal lymphadenopathy. Infants and young children under 2 years are at highest risk of developing progressive parenchymal disease and caseating lesions which may erode into pulmonary vessels, resulting in haematogenous spread to other organs or miliary TB $[9,12]$. Adult type cavitary disease is rare in children less than 10 years of age [9]. Adolescents frequently develop cavitary disease and are highly infectious [9].

As much as $25 \%$ of all TB disease in children can be extra-pulmonary [13], e.g. superficial lymphadenopathy of the cervical and submandibular chains, followed by central nervous system, bone and joint, and spinal TB. Tuberculosis meningitis can be particularly devastating because of permanent neurological sequelae.

Clinical presentation of TB in children may be vague and non-specific. Young children, especially infants, may present with fever or failure to thrive, with no respiratory symptoms. Older children may present with persistent cough and failure to thrive. Wheezing may also be a presentation when bronchial obstruction from hilar or para-tracheal 
lymphadenopathy is present. A high index of suspicion is necessary to diagnose TB in infants in highly endemic countries.

\section{The challenge of accurate diagnosis}

TB in children remains extremely difficult to diagnose in resource-poor, high-burden countries which usually also have high rates of malnutrition and HIV infection as confounding factors. Young children who are at the highest risk of TB do not expectorate sputum. Even if sputum is obtained, it is AFB smear-negative in the vast majority because children have paucibacillary disease. Symptoms and radiological findings are also nonspecific. Even in industrialised countries, the gold standard of bacteriological confirmation can only be obtained in $30-40 \%$ of children with TB, using techniques such as gastric aspirates for AFB culture [14]. In developing country settings, obtaining gastric aspirates for AFB culture, which requires hospitalisation for several days, is usually not feasible, and in practice bacteriological confirmation is rarely attempted. Instead, the diagnosis of TB in children is usually made based on history of contact with an infectious source, clinical signs and symptoms, and limited investigations such as tuberculin skin test reactivity (TST) and radiological findings. The interpretation of TST in TB endemic countries with high BCG vaccination coverage is fraught with difficulty. False negatives occur in those with the highest probability of disease - malnourished children, and children with miliary TB, or HIV/ AIDS. False positive reactions may occur in those with recent $\mathrm{BCG}$ vaccination or exposure to environmental mycobacteria. Most problematic is the lack of discrimination by TST between individuals with TB infection and those with active TB disease. Several different cut-off values for what is considered a positive TST have been advocated by different authorities [12]. For example, WHO guidelines state that a greater than $10 \mathrm{~mm}$ TST reaction should be considered positive in a BCG nonvaccinated person, and greater than $15 \mathrm{~mm}$ in a $\mathrm{BCG}$ vaccinated individual [15]. On the other hand, the American Academy of Paediatrics recommends interpretation of the TST reaction regardless of BCG vaccination status, and considers a reaction of greater than $5 \mathrm{~mm}$ as positive in any child in close contact with a case, or children clinically suspected to have TB based on signs and symptoms and xray evidence [16]. The British Thoracic Society considers $5-14 \mathrm{~mm}$ reaction as positive in a BCG non-vaccinated individuals [17].

The lack of reliable diagnostic methods and standard case definitions of childhood tuberculosis have hampered management and control of TB in children. Many attempts have been made to develop algorithmic approaches to diagnosis based on the presence of certain signs and symptoms, and other information [14]. However, most have not used appropriate control populations, and few have been properly validated against the gold standard [14]. Moreover, a gold standard based on culture confirmation is not practical for diagnosis of TB in children.

Tests that can accurately diagnose childhood TB and distinguish between TB infection and TB disease in highly endemic countries are urgently needed, if we are to control TB in children [18]. Such tests will have to be cheap and easy to perform if they are to be used in resourcepoor environments. Some immunological and cytokines based assays that measure responses to specific proteins of M. tuberculosis have shown reassuring diagnostic tests [19], but the concern is that these will be priced for marketing in industrialised countries, leaving high-burden developing countries out in the cold.

\section{Prospects for disease control}

In the long run, childhood TB can only be conquered through the use of more effective vaccines than the BCG vaccine. BCG is about $50-80 \%$ effective in preventing disseminated TB, but appears to have no protective effect against pulmonary disease [19]. Since adults with pulmonary $\mathrm{TB}$ are the main infectious reservoir the $\mathrm{BCG}$ vaccine has remained an ineffective tool in the control of childhood TB.

An understanding of TB immunology in children is critical if effective anti-TB vaccines are to be developed for use as a component in routine immunisation programmes. Several promising vaccines are currently in early stages of development. None have as yet been tested in children. Paediatricians and public health authorities need to strongly advocate for the rapid development, testing, and introduction of safe and effective vaccines targeting TB in young children.

\section{References}

1. Donald PR. Childhood tuberculosis: the hidden epidemic. International Journal of Tuberculosis and Lung Disease 2004; 8: 627-9.

2. Nelson LJ, Wells CD. Global epidemiology of childhood tuberculosis. International Journal of Tuberculosis and Lung Disease 2004; 8: 636-47.

3. Wells CD, Nelson LJ. New international efforts in childhood tuberculosis: proceedings from the 2002 Workshop on Childhood Tuberculosis, Montreal, Canada, 6-7 October 2002. International Journal of Tuberculosis and Lung Disease 2004; 8: 630-5.

4. World Health Organization. WHO Report 2002. Global Tuberculosis Control. Surveillance, Planning, Financing. WHO/CDS/TB/2002.295. Geneva, Switzerland:WHO, 2002.

5. Calder L, Hampton L, Prentice D, Reeve M. Vaughan A, et al. A school and community outbreak of tuberculosis in Auckland. New Zealand Medical Journal 2000; 113: 71-4.

6. Curtis AB, Ridzon R, Vogel R, McDonough S, Hargreaves $\mathrm{J}$, et al. Extensive transmission of Mycobacterium 
tuberculosis from a child. New England Journal of Medicine 1999; 341: 1491-5.

7. Cardona M, Bek MD, Mills K, Isaacs D, Alperstein G. Transmission of tuberculosis from a seven-year-old child in a Sydney school. Journal of Paediatrics and Child Health 1999; 35: 375-8.

8. Ewer K, Deeks J, Alvarez L, Bryant G, Waller S, et al. Comparison of T-cell-based assay tuberculin skin test for diagnosis of Mycobacterium tuberculosis infection in a school tuberculosis outbreak. Lancet 2003; 361: 1168-73.

9. Marais BJ, Gie RP, Schaaf HS, Hesseling AC, Obihara $\mathrm{CC}$, et al. The natural history of childhood intra-thoracic tuberculosis: a critical review of literature from the prechemotherapy era. International Journal of Tuberculosis and Lung Disease 2004; 8: 392-402.

10. Marais BJ, Gie RP, Schaaf HS, Hesseling AC, Obihara $\mathrm{CC}$, et al. The clinical epidemiology of childhood pulmonary tuberculosis: a critical review of literature from the pre-chemotherapy era. International Journal of Tuberculosis and Lung Disease 2004; 8: 278-85.

11. Khan E, Starke JR. Diagnosis of tuberculosis in children: increased need for better methods. Emerging Infectious Diseases 1995; 1: 115-23.

12. Shingadia D, Novelli V. Diagnosis and treatment of tuberculosis in children. Lancet Infectious Diseases 2003; 3: $624-32$.
13. Jacobs RF, Starke JR Tuberculosis in children. Medical Clinics of North America 1993; 77: 1335-51.

14. Hesseling AC, Schaaf HS, Gie RP, Starke JR, Beyers N. A critical review of diagnostic approaches used in the diagnosis of childhood tuberculosis. International Journal of Tuberculosis and Lung Disease 2002; 6: 1038-45.

15. WHO Manual. Treatment of Tuberculosis: Guidelines for National Programs. 3rd edn. Geneva, Switzerland: World Health Organization, 1997.

16. American Academy of Pediatrics. Red Book: Report of the Committee on Infectious Diseases. 26th edn. Elk Grove Village: American Academy of Pediatrics, 2003.

17. Joint Tuberculosis Committee of the British Thoracic Society. Chemotherapy and management of tuberculosis in the United Kingdom: recommendations 1998. Thorax 1998; 53: 536-48.

18. Graham SM, Gie RP, Schaaf HS, Coulter JB, Espinal MA, Beyers N. Childhood tuberculosis: clinical research needs. International Journal of Tuberculosis and Lung Disease 2004; 8: 648-57.

19. Lewinsohn DA, Gennaro ML, Scholvinck L, Lewinsohn DM. Tuberculosis immunology in children: diagnostic and therapeutic challenges and opportunities. International Journal of Tuberculosis and Lung Disease 2004; 8: 658-74.

Anita K M Zaidi, MBBS, SM, Associate Professor of Paediatrics and Microbiology, Aga Khan University, Karachi, Pakistan. Correspondence: E-mail: <Anita.zaidi@aku.edu> (Competing interests: none declared).

\section{Control of syphilis and gonorrhoea in soldiers in Egypt at the time of Napoleon Bonaparte}

Marching in the blinding sunlight also took its toll. Men developed eye disorders and acute ophthalmia, and some went blind. At Cairo, higher on the infantry's list of misfortunes were the classic scourges of syphilis and gonorrhoea. Prostitutes were numerous, and 400 of them were decapitated in an attempt to control the spread of disease. The only medical treatment was the application of mercury paste — giving rise to the saying, "One night with Venus and six with Mercury."

Thomes G Russell, Terence M Russell. Medicine in Egypt at the time of Napoleon Bonaparte. British Medical Journal 2003; 327: 1461-4. 